
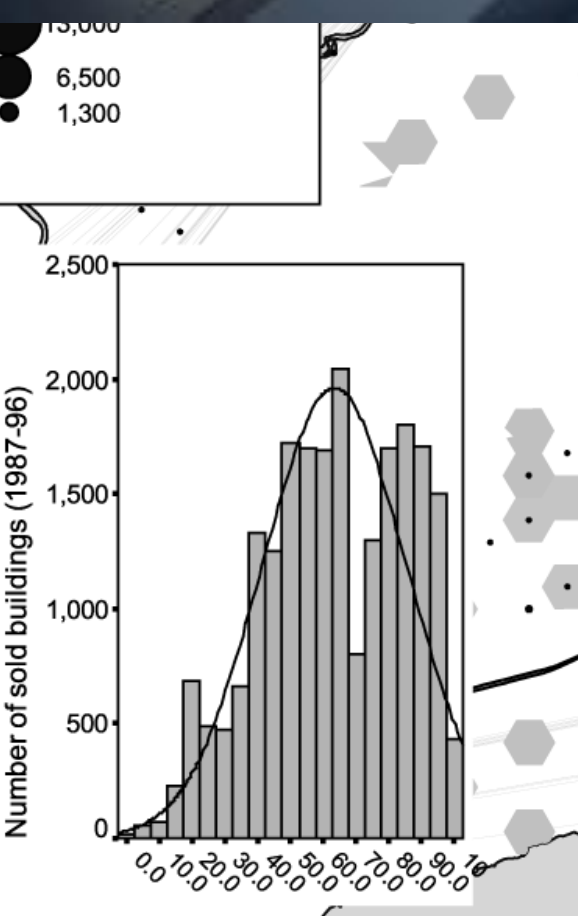

Study and Analysis of Optimized Site-selection for Urban Green Space by Using Fuzzy logic (Case Study: Seventh Region of Ahvaz Municipality) Atefeh Ahmadi, Nahid Sajadian, Hamid Jalalyian, 


\section{Study and Analysis of Optimized}

Site-selection for Urban Green Space

$$
\text { by Using Fuzzy logic }
$$

(Case Study: Seventh Region of

$$
\text { Ahvaz Municipality) }
$$

\section{Atefeh Ahmadi,}

Nahid Sajadian, Hamid Jalalyian, Seyedeh Narges Naghibirokni 


\title{
Study and Analysis of Optimized site-selection for urban green space by using Fuzzy Logic
}

\begin{abstract}
Public land-uses in many cities are not able to present desirable services to citizens because of some problems such as high population density, lack of regularity in establishment and siteselection, and lack of attention to accessibility radius and population thresholds. This problem is magnified in big cities especially for land-uses such as green spaces. In urban supplying services only increasing of services is not a reason for proper supplying services but more important is optimized distribution of these centers. In this regard, present paper based on descriptiveanalytical method as well as GIS follows optimized distribution of green space in the cities according to site-selection principals of urban green space. Case study is region 7 of Ahvaz municipality which has a population of 150215 persons and 2.78 green space capita, and this amount in addition to significant difference to standards determined by environment united nations (20-25 for a person), has been not distributed optimally. Regarding this city locating warm and dry zone of the earth, it is very important to select an optimized site for green spaces .Hence, in order to select a proper and optimized site for the green spaces according to siteselection principals layers (criteria) such as vicinity to residential centers, educational centers, cultural centers, urban infrastructures and establishments, commercial centers, hygienic centers, industrial centers and wastelands and etc. have been used. Research results in fuzzy logic valued region lands based on their importance in recognizing optimized location. Afterward, these lands were compared with land-use map and it was distinguished that lands with very good, good, and medium degree were placed close to residential, cultural, and educational centers which had more governmental ownership, and lands with weak and very weak degree were placed far from compatible land-uses, and were more placed close to industrial, administrative, hygienic sportive, and commercial centers which often had private ownership, therefore, they were not suggested for creating green space.
\end{abstract}

Index Terms - Ahvaz, Fuzzy logic, Green space, Site-selection

\section{Introduction}

Urban green space is a part of physical extent of the city which can contain specific functions. Green space in some cases has assumed aesthetic role (beautification of urban green space) and sometimes recreation role (promenade). However, as urban regions are increasing in recent decades and urbanization outshining constitution cities which has encountered different problems like immethodical increase of population, physical extension of cities causelessly, and increase in environmental pollutions, urban green space has found an important role in preserving and balancing urban environment and regulating pollution air ${ }^{[1]}$. 
In present era increasing modern urban life and culture of "curiosity of modern citizens" has resulted in increasing social relations of citizens with each other and ignoring importance of desirable public spaces for these social relations ${ }^{[2]}$. Green space connects machine sick spirit of human beings to the nature, and responds aesthetics requirements of urban population as well as fulfills expectations, hygienic, environmental, mental, and social necessities of urban society ${ }^{[3]}$. Doubtless urban green space should be considered among stable factors of natural and human life in today urbanization ${ }^{[4]}$ and if this is scheduled correctly, it will have desirable impression on treating human body and spirit ${ }^{[5]}$. Hence, if green spaces are realized properly particularly as one of environmental graces, they can be noticed as a motive for people in order to perform actions like hiking, cycling, and other recreation affairs.

As urban development consequences and complication of environmental difficulties have made existence of green space and its extension unavoidable forever, cities as the centralized focus of activity and human life have to accept structure and function affected by natural systems in order to be able to warrant their stability. Meantime, green space as an essential component of the cities plays a main role in urban metabolism, and deficiency of these spaces can create many serious disorders in urban life ${ }^{[6]}$. In this regard, public accessibility to urban services and social justice command that all urban castes can use open and green urban spaces, parks, and recreational places. That only high class of population and rich individuals of society can choose the most beautiful parts of urban landscapes for their selves to live and gradually all these landscapes belong to wealthy group of society, cannot be true ${ }^{[7]}$.

These days, attention to site-selection of urban green spaces regarding potential importance which these spaces have from the viewpoints of ecology, society, economy, mentality and so on in urban environment, is an unavoidable necessity in planning and managing the cities.

Seventh region of Ahvaz municipality with a population density of 150215 persons is counted in medium urban densities and generally used space limit of residential units and servicing components of them in medium urban densities are expressed as below:

$50 \%$ of ground surface is residential space, $25 \%$ roadway and pedestrian space, $15 \%$ green space and sportive places, and $10 \%$ other servicing spaces ${ }^{[8]}$. It should be noted that allocated capita in current state of studied region is equal to 78.2 that this amount in addition to significant difference compared with 15 capita in medium urban densities is lower than acceptable capita of green space in the cities of Iran which is 7-12 ${ }^{[9]}$ and it also has a significant distance in comparison with suggested capita in comprehensive scheme of Ahvaz which is equal to $13^{\text {[10] }}$. This research tries to achieve optimized sites for creating green spaces in studied region according to spatial analysis in GIS and fuzzy logic. Finally, the most optimized lands for creating new green space have been specified in four groups of regions with very good, good, 
weak, and very weak priority using geographical information system abilities and mentioned model.

\section{The Case Study}

Ahvaz is the center of Khuzestan province which has geographically placed between 31 degree and 20 minutes of north latitude and 48 degree and 40 minutes of east longitude in a plain with 18 meters height from the sea level ${ }^{[11]}$.Studied region in present research is region 7 of Ahvaz municipality which its area is 94000 and has a population over 150215 persons. Climate of this city is approximately dry with hot summers and temperate winters (desert climate) and its average rainfall is $250 \mathrm{~mm}$ every year. Temperature of this city ranges between 5 centigrade degree in winter and 55 centigrade degree in summer. Regarding climatic conditions of the city and its position in warm and dry region of Iran, it is essential to consider optimized and proper site-selection of urban green spaces as the recessional lungs to regulate the climate ${ }^{[12]}$.

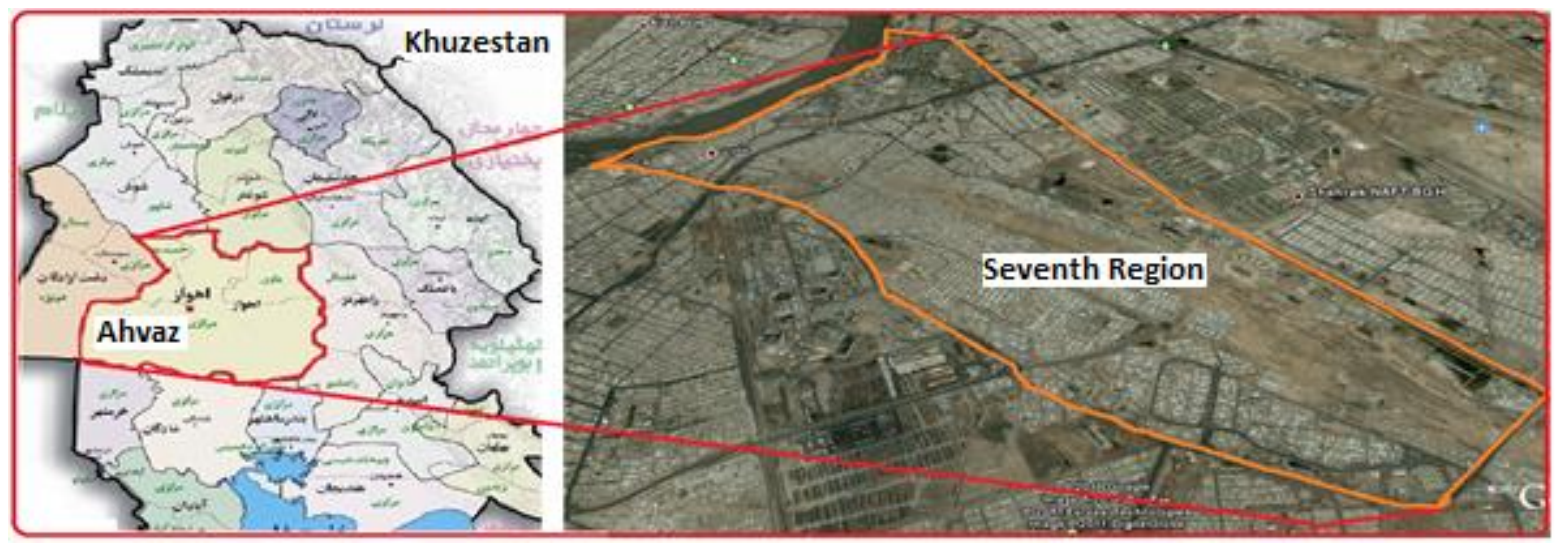

Figure 1: Position of studied region

\section{A. Methodology}

Present research is based on descriptive- analytical method and theoretic foundations have been performed according to documentary studies and field observations as well as referring to the related organizations. Criteria used for site-selection have been suggested according to siteselection principals. For selecting a proper site using geographical information system (GIS), effective factors, criteria and restrictions should be generated, processed, and analyzed in form of map layers. In other words, in execution of any project of optimized site-selection of parks in any region, it is necessary to consider different aspects such as economy, society, culture, and environment, and by regarding these proper site would be selected.

These criteria are as follows:

Regarding site-selection process is a multi-qualities decision making problem and it can be done using raster model, one important point should be considered in selecting software: selected 
software should support raster model in addition to vector model, and moreover have the abilities for using multi-qualities decision making. Therefore, in this research after creating information layers according to site-selection criteria of green space, in the next stage fuzzy logic information in ArcGIS10 software has been applied to recognize optimized site for creating green space.

Table 1: Specified standard criteria and principals for site-selection of urban green space (Resource: [13] - [14])

\begin{tabular}{ccc}
\hline Criterion & Compatibility type & $\begin{array}{c}\text { Standard distance to each land- } \\
\text { use }\end{array}$ \\
\hline $\begin{array}{c}\text { Vicinity to residential centers } \\
\text { Vicinity to educational centers } \\
\text { Vicinity to cultural centers }\end{array}$ & compatible & $\geq 150 \mathrm{Meter}$ \\
Vicinity to sportive centers & compatible & $\geq 150 \mathrm{Meter}$ \\
Vicinity to commercial centers & compatible & $\geq 150 \mathrm{Meter}$ \\
Vicinity to administrative & compatible & $\geq 150 \mathrm{Meter}$ \\
$\begin{array}{c}\text { centers } \\
\text { Vicinity to industrial centers }\end{array}$ & incompatible & $\geq 150 \mathrm{Meter}$ \\
$\quad \begin{array}{l}\text { Vicinity to urban } \\
\text { infrastructures }\end{array}$ & incompatible & \\
Vicinity to hygienic centers & incompatible & $500-1000 \mathrm{Meter}$ \\
Vicinity to wastelands & incompatible & $150-500 \mathrm{Meter}$ \\
\hline
\end{tabular}

\section{B. Preparing layers to GIS}

At this stage data in form of usable map layers in GIS environment was converted in order to be analyzed and site-selection missions be performed. After creating different information layers, map layers used in GIS environment converted to analyze and perform normalization operations and model execution. This stage contains a process in which data receive, format changes, georeferencing, calibrating and data documentation occur ${ }^{[15]}$. Data used in geographical information system include digital map of current land-use and digital maps of layers including residential, educational, cultural, sportive, administrative, industrial, and military centers. By using current data new layers were obtained such as distance from residential centers, distance from cultural centers, distance from wastelands, distance from commercial centers, distance from industrial centers, distance from urban infrastructures, distance from remedial centers, distance from administrative centers, distance from hygienic centers, and distance from sportive centers as shown in Figure - 2 (maps 1-10). 
Figure 1: distance from administrative centers

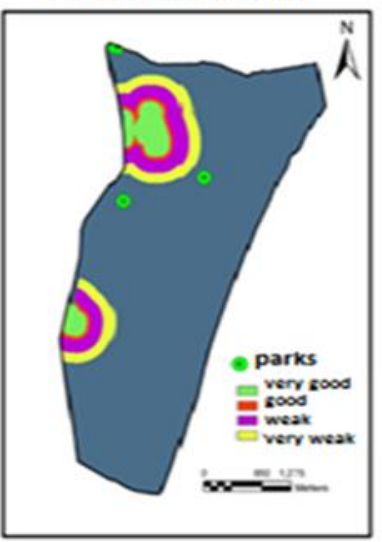

Figure 2: ditance from educational centers

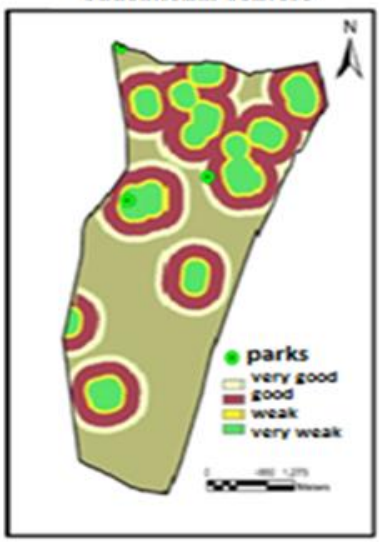

Figure 3: distance from

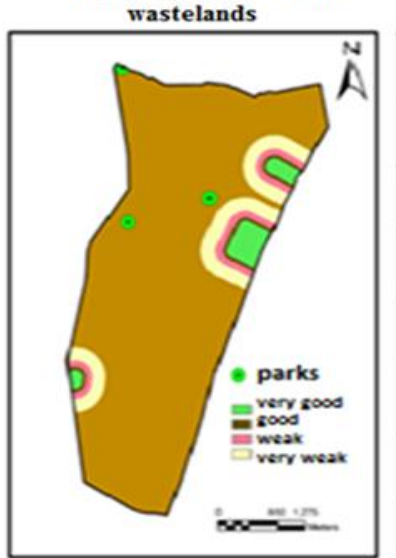

Figure 4: ditance from sporty centers

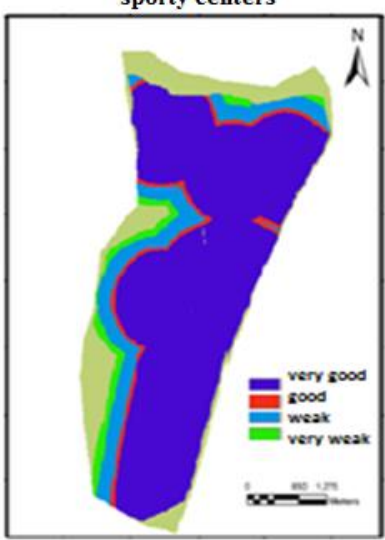

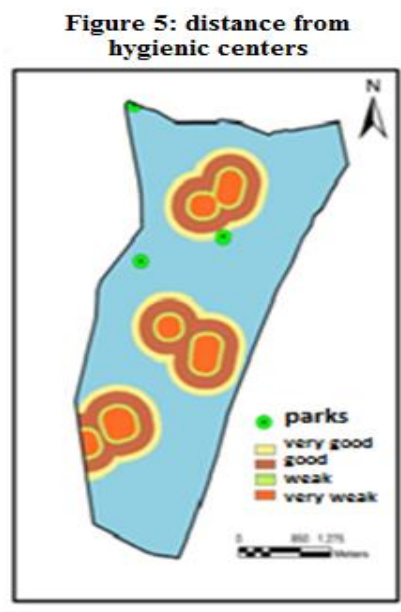

Figure 6: distance from urban infrastructures

Figure 7: distance from

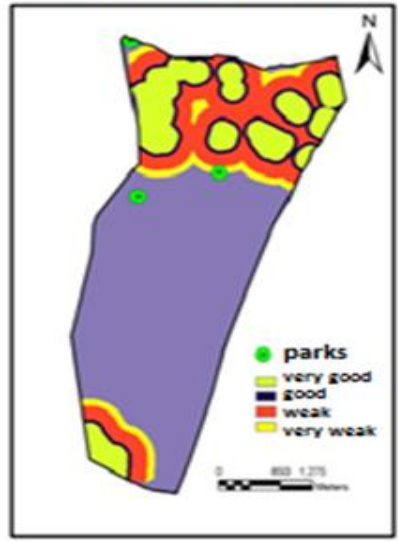
commercial centers

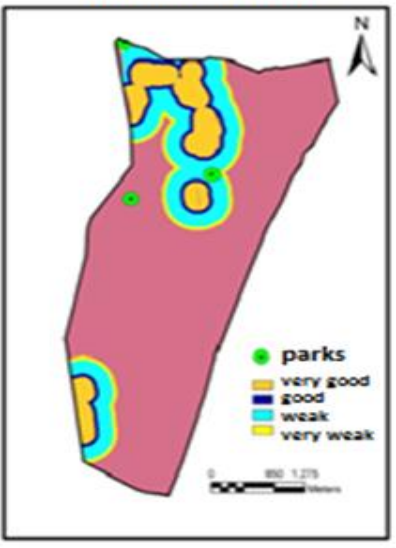

Figure 8: distance from residentail centers

$$
\begin{aligned}
& \text { Figure 9: distance from } \\
& \text { cultural centers }
\end{aligned}
$$
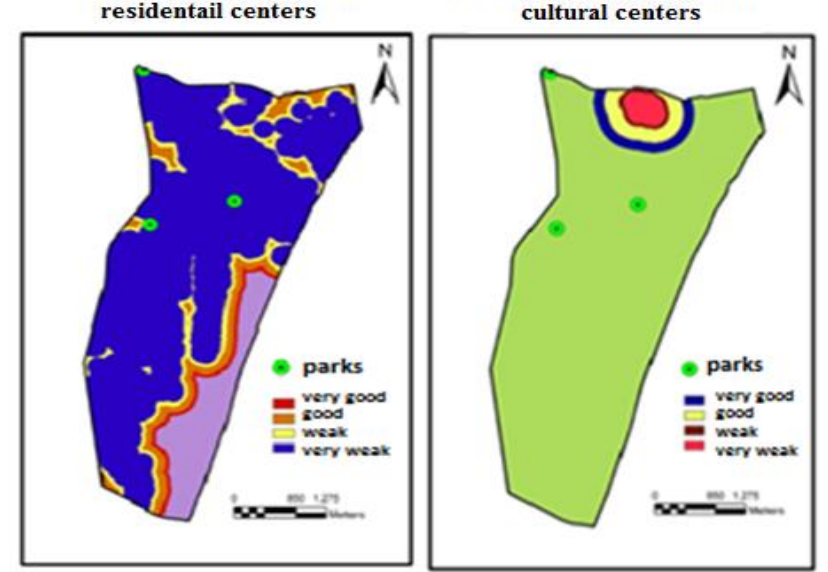

Figure 10: distance from indutreial centers

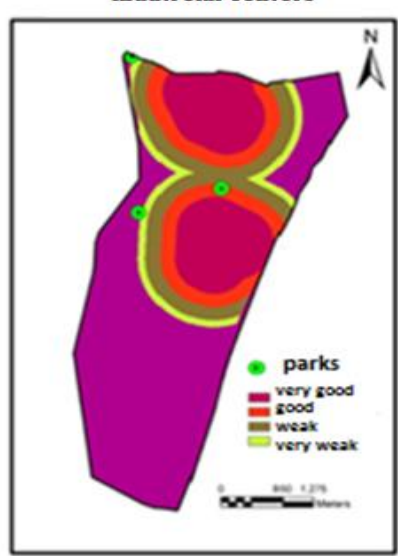

Figure 2: Distance maps from different land-uses 


\section{Normalization of maps in Fuzzy Logic}

In fuzzy logic, each area regarding the degree considered by each criterion (X), gives a membership degree $(\mu \mathrm{x})$ which expresses desirability amount of that area. It means that each area with higher membership degree has higher desirability. In this logic each layer places in a scale between 0 and 1 or a fraction of one. In these scales the larger numbers are, the more desirability they will have. It means number one has the highest desirability and number zero has not any desirability, and the range of numbers placed between these two amounts and as they become close to one the desirability will increase. In addition to selection of scale to generate fuzzy maps, type of fuzzy function should be surveyed and more desirable function should be selected for regarded criterion. Among famous functions it can indicated Large, Small, Near, Gusian, Linear, and J-Shape functions ${ }^{[16]}$. Mentioned functions are in ArcGIS and RDRIS environment and users in addition to these functions can definite their own function regarding their need (Figure - 3, A, B, and C). (In present research criterion maps have been normalized using these functions in ArcGIS10 software and their values converted into comparable units from 0 to 1).

The very important point in this step is considering selection of function type. Regarding normalization layers performed for compatible and incompatible land-uses in this research, type of selected function for instance in compatible land-uses-as these land-uses place in a close distance to green space their desirability will increase- should be defined based on performed classification and site-selection principals that classes with low distance will receive more value. Therefore this definition is harmonized with Small function. Similar way is also applied for the incompatible land-uses. As green space land-use locates in a far distance of these land-uses it will be more desirable.

Hence, classes with high distance in these land-uses based on performed classification will receive high value, and it is harmonized with Large function. Moreover, one of effective factors in normalization of fuzzy maps is demarcating threshold which is also called control points. Another point which should be regarded in selecting function is its decreasing and increasing type ${ }^{[17]}$. Decreasing function means becoming minimum or decreasing and increasing function means becoming maximum or increasing. For instance, as for the distance from commercial centers, the more distance it is, the better it will be. As a result, here we will use increasing function. Table 2 has demonstrated threshold amounts and fuzzy function type in order to normalize criterion maps in fuzzy logic.

Finally, after normalization of layers and execution of proper functions for each layer all normalized layers were integrated in fuzzy overlay according to harmonized function and at the end lands with priority were recognized for creating green space (Figure -4 ). 

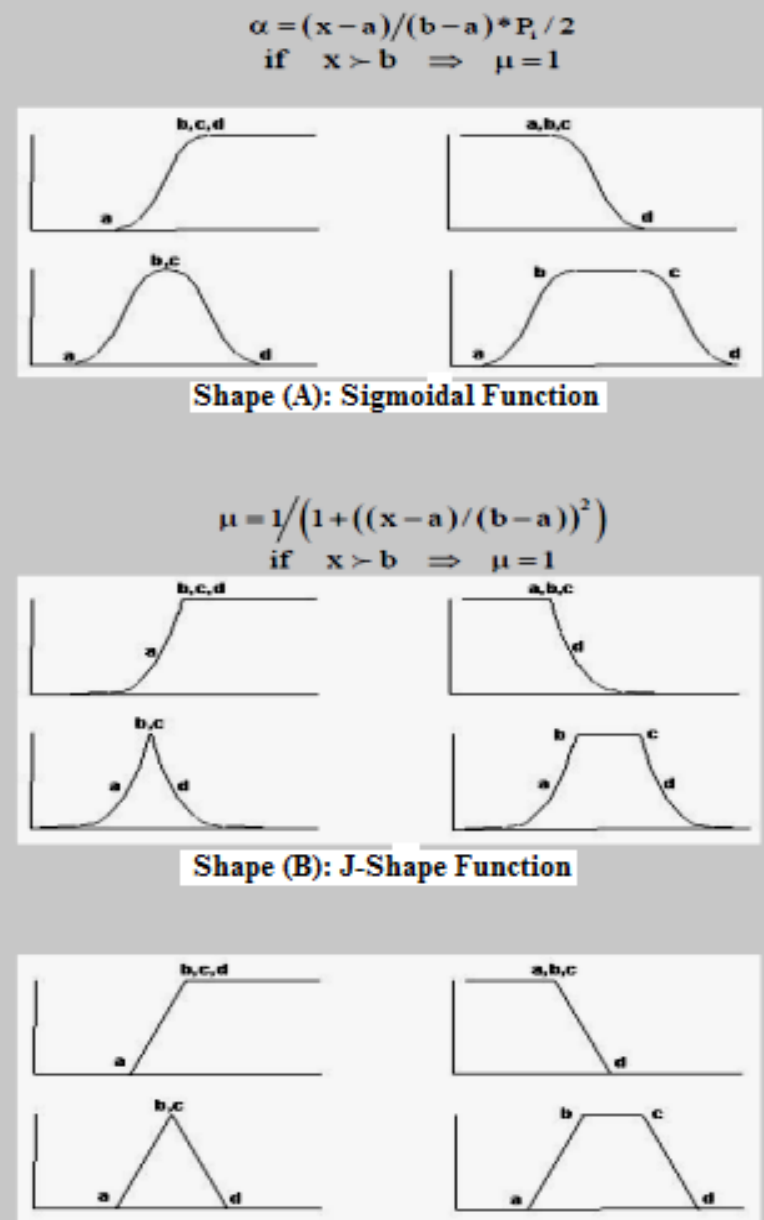

Shape (C): Linear Function

Figure 3: A: Sigmoidal function, B: J-shape function, C: Linear function 
Table 2: Threshold and fuzzy function type to normalize criterion maps in fuzzy logic

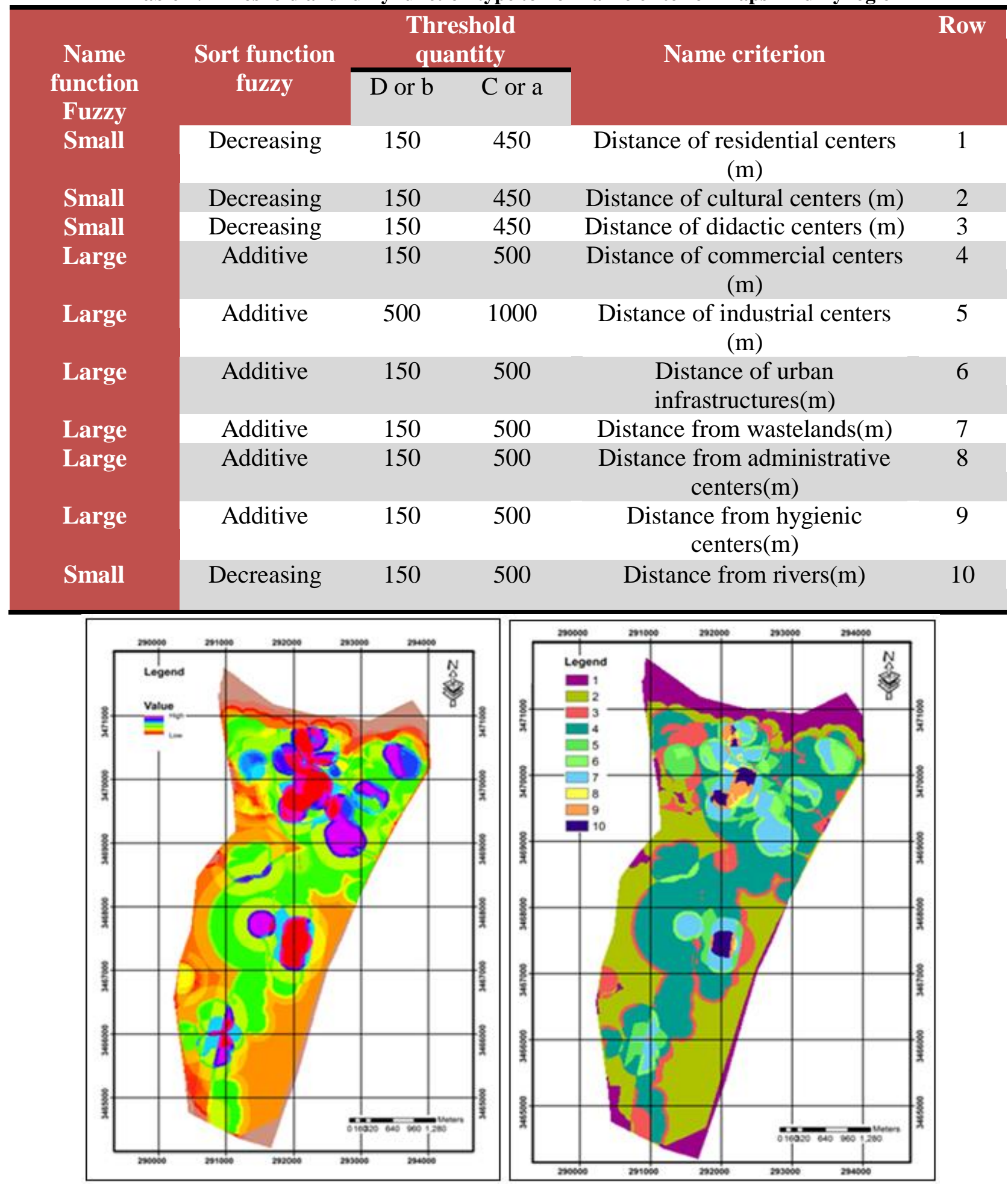

Figure 4: $\quad$ Part A - Final integration after performance functions in fuzzy logic. Part B - Valuation of urban lands for creating green space by fuzzy logic 


\section{Conclusion}

Emersion of environmental crisis in cities and reduction of public level life have been made treatment of urban environment and preservation of it important for future generation. Hence, in spatial development programs it is important to consider green space in order to achieve balanced, equal, and sustainable development. In present time, environmental pollutions in most cities are increasing, so harmonized and equal distribution of parks and urban green spaces plays an effective role in creating environmental stability of cities.

In this research site-selection of urban green spaces using fuzzy logic was studied to present optimized pattern and effective park distribution of region 7 in Ahvaz city. Results derived from final output layer of this model in ArcGIS10 and its adaption with land-use map of Ahvaz city indicates that regions with very good degree are close to residential, educational, and cultural centers and have distance from incompatible land-uses and radius function of current parks and many governmental centers (such as educational, administrative centers and etc.) are located in this area. Hence, by changing their land-use and converting their ownership into green space, the problems of current lands will be reduced. Lands with good and medium degree in studied region are close to educational, residential, and cultural centers. They are also further from radius function, and their ownership is governmental, so if the land-use changes, there will not create many problems. As for the lands with weak degree, these lands on one hand due to locating close to incompatible land-uses like industrial, hygienic, administrative, and commercial centers and on the other hand due to have private ownership, changing their ownership will face them with many problems. Therefore, these lads are not suggested for creating green space. Another type of lands with priority for green space land-use contains lands with very weak degree. These lands were not suggested for creating green space due to vicinity to radius function of current parks and far distance from residential, educational, and cultural centers as well as locating in functional area of incompatible infrastructures. Results derived from this research demonstrated application and importance of fuzzy logic in site-selection of green space and presented optimized pattern distribution according to population needs and principals.

\section{References}

1. J. Mohammadi, "Geographical information system in urban green space site-selection," Shahrdariha journal, no .44, pp. 15, 2001.

2. K. Suzanchi, and F. Behforuz, "Technical office of harmony assistance of reclamation affairs," Iran ministry publications, pp. 5, 2001.

3. A. Pur Ahmad, A. Pursaraskanrud and M. Sotudeh, "Urban green space management of Tehran municipality 9th region, Human Geography Researches, no. 69, year. 41, pp. 31, 2009. 
4. A. Esmaeeli, "Analysis of urban parks from viewpoint of urban planning approach (case study: region 1 and 8 of Tabriz)," M.S. thesis, Univ., Tarbiat Modares, Tehran, Iran, p. $11,2003$.

5. Shiri, "Presentation of optimized site-selection of urban green space by using GIS, case study: urban parks of Zanjan,” M.S. thesis,Univ., Zanjan, Iran, p. 32, 2006.

6. H. Majnunian, "Arguments surrounding parks, green space, and promenades," urban service assistance of park and green space, Tehran, summer, p. 6, 1995.

7. E. Zangiabidi and Mokhtarie malekabadi, "Cities, green space, and new approach to human design dimensions," Payame sabz journal, no. 42, 2005.

8. E. Shieh, "An introduction on urban planning principals, science and industry university publications, Tehran, Iran, no. 67, pp. 173-179, 1990.

9. A. Saeednia, Green book municipality, Studies and urban planning center, Iran ministry publications, vol. 9, p. 83, 2000.

10. Housing and urbanization,comprehensive scheme Ahvaz, Iran, 2006.

11. Programming assistance of Khuzestan governor, Khuzestan province yearbook, census and information of Iran ministry, 2006.

12. A. Ahmadi, A. Movahed, A Shojaian, "Presentation of optimized site-selection of urban green space by using GIS (case study: 7th region of Ahvaz municipality)", scientific and research journal of environmental preparation, no. 15, Malayer university, winter, p.6, 2011.

13. S. A. Hossaini, "Study of educational land-uses in Tehran city and presenting proper pattern,” M.S. thesis, Univ., Taarbiat Modarres, Tehran, Iran, p. 41, 2001.

14. H. R. Varesi," Urban green space site-selection by using geographical information system (case study: Korram abad city)," Geography and regional planning journal, no. 10, Spring and Summer, p. 93, 2008.

15. M. Farajzadehasl, and S. Hushang, "Management and site-selection of educational centers by using geographical information system," geographical researches journal, no. 67, p. 8, 2002.

16. Eastman, J.R. IDRISI for windows users guide, version 3.2, Clark labs for cartographic technology and Geographic Analysis, Clark University, 1997. 
17. K. Valizadeh and H. Shahabi, "Necessities of GIS Usage in urban water management At The Time of Natural Accidents (Case Study: Saghez City)," International Conference on Geographic, Paris, France, 2009.

18. A. Ahmadi, "Tourists optimal path-finding by GIS (Case study: historical texture of Sanandaj)", 5 th Symposium on Advances in Science \& Technology, 5thSASTech ,May, Mashhad, Iran, pp. 12-17, 2011.

19. H. Shahabi, "The role of geomorphic factors in site-finding of urban waste burial in Saghez using GIS models and remote sensing technology," M.S. thesis, Univ., Tabriz, Iran, 2009.

- END - 


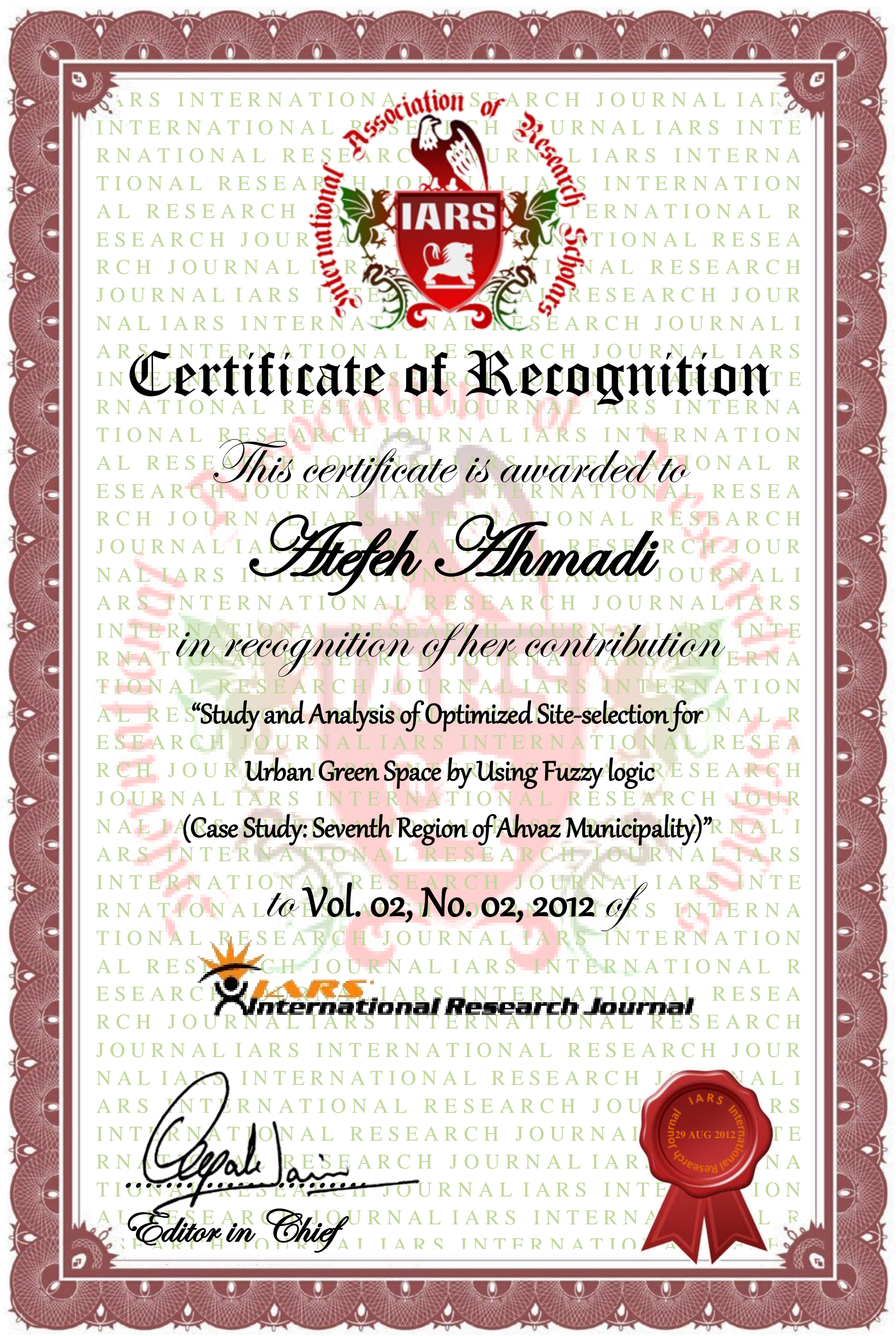

